\title{
Preliminary Guidelines for the Use of IVIg during COVID-19
}

\section{David S. Younger}

School of Medicine, Department of Medicine, Division of Neuroscience, Graduate School of Public Health, Department of Health Policy and Management, City University of New York (CUNY), New York, USA

Email: youngd01@nyu.edu

How to cite this paper: Younger, D.S. (2021) Preliminary Guidelines for the Use of IVIg during COVID-19. World Journal of Neuroscience, 11, 211-220.

https://doi.org/10.4236/wjns.2021.112015

Received: April 28, 2021

Accepted: May 28, 2021

Published: May 31, 2021

Copyright (c) 2021 by author(s) and Scientific Research Publishing Inc. This work is licensed under the Creative Commons Attribution International License (CC BY 4.0).

http://creativecommons.org/licenses/by/4.0/

\begin{abstract}
Infection with the severe acute respiratory syndrome novel type-2 novel coronavirus (SARS-CoV-2) responsible for the 2019 coronavirus disease (COVID-19) shows a highly heterogeneous clinical presentation and age affliction in children and adults, ranging from asymptomatic or mild disease to severe involvement, with potentially fatal respiratory failure and multiple organ dysfunction. As susceptibility to severe COVID-19 depends upon comorbid factors including immune competence, optimizing the latter through low-dose supplementation or high dose treatment with immune globulin therapy in those with primary immune deficiency and post-infectious immune sequelae of SARS-CoV-2 and existing autoimmune disorders is essential. There are no existing guidelines hence; this paper provides a framework for considering preliminary guidelines for the use of immune globulin therapy during COVID-19.
\end{abstract}

\section{Keywords}

SARS-CoV-2, Immune Globulin, Neurological, Nervous System, Post-Acute Sequelae, COVID-19

\section{Background and Rationale}

SARS-CoV-2 infection triggers a dysregulated innate and adaptive immune response accompanied by hyperinflammation known as the cytokine storm [1] during which the viral escapes from innate sensing particularly the interferon I (IFN-I) pathway, with activation of myeloid cells, depletion of T-cells, NK T-cell dysfunction, and hemostatic imbalance with widespread clotting. The most dismal outcome is observed in older patients with excessive comorbid disease [2] who sustain hypoxic and inflammatory brainstem and cerebral insults before 
succumbing to pulmonary embolism, multiple organ failure, and fatal cardiopulmonary arrest [3], and children affected by multisystem inflammatory syndrome (MIS-C) [4]. Unvaccinated younger adults, ages 18 to 23 years [5] and very young school-aged children, ineligible for vaccination who, promote community spread [6] may suffer worse COVID-19 outcomes when they have at least one underlying medical condition [7]. Other children with established pediatric autoimmune neuropsychiatric disease associated with Group A beta hemolytic streptococcus (PANDAS) and the closely related disorder PANS, triggered by other microbial agents, notably autoantibody-positive and seronegative autoimmune encephalitides (AE) [8] may be vulnerable to post-infectious SARSCoV-2 hyperimmunity. Clinicians treating severe hospitalized COVID-19 may administer monthly high-dose intravenous immune globulin (IVIg-HD) therapy at doses of $1-2$ grams per kilogram $(\mathrm{g} / \mathrm{kg})$ over 3 to 5 days to delay progression and improve mortality of SARS-CoV-2-related illness [9]. However, IVIg-HD therapy may be safely administered and well-tolerated at higher total monthly doses with careful monitoring of serum IgG levels, and renal, liver, bone marrow and clotting parameters. More recently, IVIg-HD has been used in the empiric treatment of post-acute sequela of COVID-19 (PASC) [10]. This disorder is defined by the National Institutes of Health [11] as new or persistent organ-related symptoms lasting longer than one month after initial infection. Vulnerable individuals including those ineligible or hesitant to vaccinate, and pre-existing immune deficiency or autoimmune illness, as well as others recovering from SARSCoV-2-related exposure with acquired immune deficiencies, may benefit from monthly low-dose (LD) (400 - $500 \mathrm{mg} / \mathrm{kg}$ ) (IVIg-LD) or standard subcutaneous (SC) Ig therapy to restore normal host immunity [12]. Table 1 shows some of the neurological disorders in which Ig therapy has had a treatment role during COVID-19.

The way forward for prospective candidates benefitting from Ig therapy during the COVID-19 pandemic and afterward remains to be charted. This article provides a series of recommendations for clinicians, patients, and lay groups on this subject.

\section{Literature Search Methodology}

The search rationale followed a PICO model including children and adults (P) treated with Ig (I): LD or HD and SC or IV for post-infectious SARS-CoV-2-related or incidental immune disorders (C), and the immune modulatory outcome (O) thereof.

\subsection{Limitations}

There is a paucity of literature on the judicious use of IVIg during COVID-19 and in response to the post-infectious sequelae thereof. Furthermore, the proposed guidelines are not intended to be reflective of its use over or above other established immune modulatory therapies. 
Table 1. Adult and pediatric neurological disorders suggested for use of Ig therapy during COVID-19.

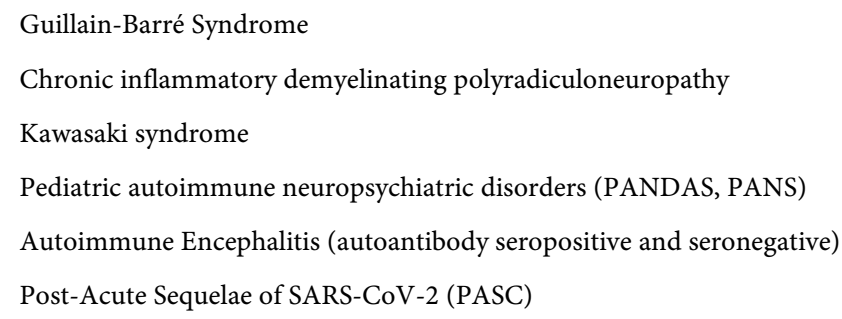

\subsection{Severe COVID-19}

Three hundred seventeen (317) reported patients received IVIg therapy for severe hospitalized COVID-19 in 2020, including 174 patients in a multi-center study [13], 119 patients in three single center studies [14] [15] [16], eight patients in two cases series (8 patients) [17] [18], and 17 patients in an open-label randomized clinical trial [19]. Doses ranged from 0.1 to $0.5 \mathrm{~g} / \mathrm{kg}$ for 3 to 5 days over a month, generally in association with the SOC that included corticosteroids (CS) and antibiotics, antivirals, or interferon therapy. Primary endpoints of reduced 28-day, 30-day and 60-day mortality and prevention or slowing of progression were cited as favorably influenced by the addition of IVIg to the SOC. Four clinical trials studying IVIg in severe COVID-19 were listed in ClinicalTrials.gov as not yet recruiting (NCT04616001, NCT04411667), recruiting (NCT04480424), or completed (NCT04400058).

The benefit of IVIg in severe and critically ill COVID-19 is not well understood, however several studies suggest that it targets the post-infectious inflammatory response of the cytokine storm by scavenging complement, regulating effector T-helper (Th) 1 and Th17 cells, and regulatory T-cells while inhibiting host innate immune cell activation and secretion of inflammatory mediators. Its action to reduce elevated plasma levels of IL-6 and C-reactive protein (CRP) and other laboratory markers of inflammation early in the course of COVID-19 illness is consistent with downregulation of the immune response. While current batches of IVIg may neutralize seasonal coronavirus species, it generally lacks cross-neutralizing antibodies to SARS-CoV-2 [20]. Although passive virus neutralization appears to not be responsible for the beneficial effects of IVIg in treating COVID-19, SARS-CoV-2 encodes one superantigen motif near its S1/S2 cleavage site that might trigger the cytokine storm [21]. As IVIg contains antibodies reactive against SARS-CoV-2 antigens [22], IVIg might inhibit superantigen-mediated T-cell activation, cytokine release and an incipient cytokine storm.

\section{Adult and Childhood Post-Acute Sequelae of SARS-COV-2 (PASC)}

The scientific community and healthcare professionals not only face the dilemma of understanding the diverse ways in which SARS-CoV-2 affects the host cells, but also the capability of the virus to exert prolonged organ effects espe- 
cially in the nervous system, after the acute-phase of infection. Affected patients may fail to revert to normal and instead develop new organ symptoms, especially in the nervous system. It is unlikely that the proposed designations, "Covid Long Hauler (LH) and Long Covid" [23] are inclusive or accurate enough to describe this disorder. However, given the magnitude of those affected, and their congregation in self-selected social media and Facebook groups, they may be useful cohorts for future studies. Instead, through its recent launch by the NIH of the SARS-CoV-2 Recovery Cohort and Investigator Consortium [11], it focuses attention on PASC and LHs.

Patient led research stemming from online surveys provides some useful information. Davis and colleagues [24] characterized the symptom profile and time course in 3762 patients with Long COVID, along with its impact on daily life, work, and return to baseline health using an international web-based survey design of suspected and confirmed COVID-19 cases with illness lasting from 28 days to 6 months from mid- to late 2020. The most likely symptoms to persist were fatigue, post-exertional malaise, cognitive dysfunction ("brain fog"), neurologic sensations (neuralgias, weakness, coldness, electric shock sensations, facial paralysis/pressure/numbness), headaches, insomnia, shortness of breath, postural dizziness, lightheadedness, and tachycardia suggesting tandem central, peripheral, and autonomic nervous system (CNS, PNS and ANS) involvement. Similar findings have been reported by other investigators [25] [26].

Younger [10] described progressive CNS, PNS and ANS involvement that developed in a patient convalescing from COVID-19 illness prompting treatment with IVIg. A mechanism of post-infectious immunity, which appeared likely in this patient and in other LHs, is best described by the acronym, I-Cubed $\left(\mathrm{I}^{3}\right)$, that posits the multiplier effect of infection, immunity, and inflammation, conditioned by environmental and genetic predisposing factors, as the source of nervous system autoimmunity [27]. While awaiting the results of large-scale cohort studies from the NIH, IVIg is first-line recommended treatment for adults and children with PASC.

The MIS-C and COVID-19-Related Hyperinflammation Task Force of the American College of Rheumatology studying MIS-C [28] has addressed treatment of children with COVID-19 related hyperinflammation and MIS-C. MIS-C is a disorder that resembles Kawasaki Disease (KD) both of which have a potentially fatal outcome due to cardiac and nervous system damage related to systemic vasculitis if not treated promptly with IVIg therapy. As in KD [29], the prompt administration of IVIg-HD in MIS-C may be life-saving. The optimal monthly dose of IVIg-HD should be guided by the achieved clinical response, and may safely exceed $2 \mathrm{~g} / \mathrm{kg}$ in a given month. Given the safety profile of IVIg, there is implicitly little rationale for withholding a second $2 \mathrm{~g} / \mathrm{kg}$ dose in one month. Such an empiric approach has been extrapolated to AE, PANDAS/PANS and other autoimmune encephalopathies wherein remission status relates to baseline IgG deficiency [30], and the risk-benefit ratio may favor the timely ad- 
ministration of one or more induction IVIg-HDs in a single dose with careful monitoring before commencing maintenance IVIg therapy of $1 \mathrm{~g} / \mathrm{kg}$ every 3-4 weeks.

\section{Genetic and Acquired Immune Deficiency}

IVIg was first employed to treat immunodeficiencies in the 1950s and is currently licensed for the treatment of primary immunodeficiencies (PIDs) with impaired antibody production, secondary immunodeficiencies with recurrent infections, selective $\operatorname{IgG}$ and $\operatorname{IgA}$ antibody deficiencies, and proven specific antibody failure (PSAF) [31]. As a group, selective or pan-antibody deficiencies represent the most common types of PID. Symptoms often do not appear until the latter part of the first year of life, as passively acquired IgG from the mother decreases to below protective levels. The spectrum of antibody deficiencies is broad, ranging from the most severe childhood (X-linked agammaglobulinemia) to decreased serum levels of IgG and IgA, with elevated levels of IgM (HIGM syndrome). All manifest recurrent sinopulmonary and gastrointestinal bacterial infections.

Adults with common variable immunodeficiency (CVID) commonly present with recurrent otitis media, chronic sinusitis, and recurrent pneumonia. Selective IgA deficiency is usually accompanied by acquired or genetically reduced IgA1 or 2 subclass levels. IgA-deficient patients at risk for the development of anti-IgA antibodies on receipt of IVIg may experience anaphylaxis. IgG subclass deficiencies are common in adults and children as single or multiple class deficiencies.

Shields and coworkers [32] studied the outcome of 100 COVID-19 with PID and secondary immunodeficiency (SID) in the Immunodeficiency Network cohort noting greater morbidity and mortality compared to the general population. CVID was the most common PID with an infection-fatality ratio (IFR) of $20.0 \%$ (12 of 60 ) in a population of median age 42.0 years, with an even poorer outcome of PID noting an IFR of $33.3 \%$ (11 of 33) in a population of median age 64.5 years the most common causes of which were hematologic and lymphoproliferative cancers, compared to $14.3 \%$ and $1.5 \%$ at the same time-period from May to August 2020.

The management of PID and SID includes IVIg therapy to restore serum Ig levels and humoral immunity back to normal. The comparatively high morbidity and mortality in PID and SID should inform public health policy and be communicated to affected and at-risk patients so they can take appropriate actions to reduce their exposure to the virus and have access to IVIg therapy. It remains to be seen whether patients with PID and SID elaborate normal amounts of SARSCoV-2 IgG and if IVIg prevents or increases that likelihood.

\section{Autoimmunity}

It is not known whether incidental treatment of autoimmune diseases (AD) with 
IVIg prevents or reduces infection rate or severity of COVID-19 illness, an anecdotal prospective analysis of 55 children and adults treated with IVIg-HD or LD therapy for diverse acquired and post-infectious autoimmune neurological disorders found no new cases of SARS-CoV-2 stratified by a single home infusion service via phone interviews at the height of the Covid-19 pandemic (when it would have been impermissible for a nurse to enter the home [33]. This uncontrolled observation suggests that IVIg therapy may have an important role in COVID-19 illness prevention among vulnerable individuals with established ADs. However, a longer period of follow-up of this cohort will be necessary to confirm these observations, as are further controlled studies to identify the dose and frequency of IVIg treatment to confer prophylactic efficacy. An unpublished larger experience among 1000 similarly treated subjects found 10 instances of self-reported COVID-19 illness interrupting IVIg treatment indicating a much lower infection rate of 0.01 .

Neither has it been established whether SARS-CoV-2 leads to frank autoimmune illness like other viruses that exhibit molecular mimicry, bystander activation, epitope spreading and other established mechanisms of post-infectious autoinflammatory immune responses [34] [35] as in the examples of enteric virus-associated type I diabetes (T1D), hepatitis C virus-related cryoglobulinemic vasculitis, and post-influenza acute disseminated encephalomyelitis (ADE).

However, experts agree that from a pathogenesis standpoint, SARS-CoV-2 triggers a vigorous host immune response that appears to be not only crucial for viral clearance, involving both the innate and adaptive immune arms, but also immune changes responsible for post-acute sequelae and long-lasting autoimmune diseases (AD) [36]. However, SARS-CoV-2 is linked to many autoinflammatory disease states that emerge in acute COVID-19 illness, even before they can be properly labeled $\mathrm{AD}$, and benefit from IVIg including Guillain-Barré syndrome (GBS) [37], KD [38] and thrombotic microangiopathy (TMA) with abnormal complement activation [39]. Large epidemiological studies involving case-control studies and prospective cohorts treated with IVIg in the course of COVID-19 illness may illuminate whether it impacts autoinflammatory and AD sequela.

A very recent publication in press at the time of this writing [40] demonstrates PASC in a child with striking similarity to the adult conditionclinically and in its responsiveness to HD-IVIg suggesting a continuum of potentially prolonged post-COVID-19 neurological autoimmunity in the absence of effective treatment.

\section{Conclusion}

It is a public health priority to better understand and develop strategies to prevent and treat the post-acute sequelae of SARS-CoV-2 infection (PASC). IVIg is a well-established immunomodulatory therapy with the potential to impact COVID-19 illness throughout the timeline of the infectious and post-infectious 
autoimmune process.

\section{Recommendations}

While awaiting the results of the NIH sponsored SARS-CoV-2 Recovery Cohort [10], some useful guidelines can be posited in the use of IVIg.

1) IVIg-HD is indicated in all patients acute severe COVID-19 to ameliorate the post-infectious autoimmune cytokine storm and widespread clotting due to complement-mediated TMA.

2) IVIg-HD is indicated in all patients COVID-19 who present with or develop autoimmune disorders such as GBS who would normally be treated in the absence of COVID-19.

3) IVIg-HD is indicated in all children with MIS-C especially severe cases resembling KD.

4) IVIg-LD is indicated in all unvaccinated patients with autoimmune disorders to provide immune support.

5) IVIg-LD is indicated in all patients with PID and SID for protection against COVID-19 exposure.

6) IVIg-HD is indicated in patients with PID and SID who succumb to acute COVID-19 to avert further complications including the cytokine storm and TMA.

7) IVIg is indicated therapy in several neurological disorders with an etiopathogenesis related to the post-infectious autoimmune sequela of COVID-19.

Readers are referred to https://www.davidsyounger.com for recommendation updates.

\section{Conflicts of Interest}

The author declares no conflicts of interest regarding the publication of this paper.

\section{References}

[1] Caricchio, R., Gallucci, M., Dass, C., Zhang, X., Gallucci, S., Fleece, D., et al. (2021) Preliminary Predictive Criteria for COVID-19 Cytokine Storm. Annals of the Rheumatic Diseases, 80, 88-95. https://doi.org/10.1136/annrheumdis-2020-218323

[2] Lithander, F.E., Neumann, S., Tenison, E., Lloyd, K., Welsh, T.J., Rodrigues, J.C.L., et al. (2020) COVID-19 in Older People: A Rapid Clinical Review. Age and Ageing, 49, 501-515. https://doi.org/10.1093/ageing/afaa093

[3] Younger, D.S. (2021) Postmortem Neuropathology in COVID-19. Brain Pathology, 31, 385-386. https://doi.org/10.1111/bpa.12915

[4] Kabeerdoss, J., Pilania, R.K., Karkhele, R., Sathish Kumar, T., Danda, D. and Singh, S. (2021) Severe COVID-19, Multisystem Inflammatory Syndrome in Children, and Kawasaki Disease: Immunological Mechanisms, Clinical Manifestations and Management. Rheumatology International, 41, 19-32. https://doi.org/10.1007/s00296-020-04749-4

[5] Wilson, R.F., Sharma, A.J., Schluechtermann, S., Currie, D.W., Mangan, J., Kaplan, B., et al. (2020) Factors Influencing Risk for COVID-19 Exposure among Young 
Adults Aged 18-23 Years-Winnebago County, Wisconsin, March-July 2020. Morbidity and Mortality Weekly Report, 69, 1497-1502.

https://doi.org/10.15585/mmwr.mm6941e2

[6] Rankin, D.A., Talj, R., Howard, L.M. and Halasa, N.B. (2021) Epidemiologic Trends and Characteristics of SARS-CoV-2 Infections among Children in the United States. Current Opinion in Pediatrics, 33, 114-121.

https://doi.org/10.1097/MOP.0000000000000971

[7] Leeb, R.T., Price, S., Sliwa, S., Kimball, A., Kimball, A., Szucs, L., Caruso, E., et al. (2020) Trends among School-Aged Children-United States, March 1-September 19, 2020. Morbidity and Mortality Weekly Report, 69, 1410-1415.

https://doi.org/10.15585/mmwr.mm6939e2

[8] Younger, D.S. (2019) Autoimmune Encephalitides. Neurologic Clinics, 37, 359-381. https://doi.org/10.1016/j.ncl.2019.01.015

[9] Galeotti, C., Kaveri, S.V. and Bayry, J. (2020) Intravenous Immunoglobulin Immunotherapy for Coronavirus Disease-19 (COVID-19). Clinical \& Translational Immunology, 9, e1198. https://doi.org/10.1002/cti2.1198

[10] Younger, D.S. (2021) Post-Acute Sequelae of SARS-CoV-2 Infection Associating Peripheral, Autonomic, and Central Nervous System Disturbances. Case Report and Review of the Literature. World Journal of Neuroscience, 11, 17-21. https://doi.org/10.4236/wjns.2021.111003

[11] National Institutes of Health (2021) Post-Acute Sequelae of SARS-CoV-2 Infection Initiative: SARS-CoV-2 Recovery Cohort Studies. No. OTA-21-015B. National Institutes of Health, Bethesda.

https://covid19.nih.gov/sites/default/files/2021-02/PASC-ROA-OTA-Recovery-Coh ort-Studies.pdf

[12] Younger, D.S. (2020) Immunotherapy for the Post-Infectious Sequela of SARSCOV-2 Infection. World Journal of Neuroscience, 10, 117-120.

[13] Shao, Z., Feng, Y., Zhong, L., Xie, Q., Lei, M., Liu, Z., et al. (2020) Clinical Efficacy of Intravenous Immunoglobulin Therapy in Critical Ill Patients with COVID-19: A Multicenter Retrospective Cohort Study. Clinical \& Translational Immunology, 9, e1192. https://doi.org/10.1002/cti2.1192

[14] Zhou, Z. G., Xie, S. M., Zhang, J., Zheng, F., Jiang, D., Li, K., et al. (2020) Short-Term Moderate-Dose Corticosteroid plus Immunoglobulin Effectively Reverses COVID-19 Patients Who Have Failed Low-Dose Therapy. Preprints, 2020, Article ID: 2020030065. https://doi.org/10.20944/preprints202003.0065.v1

[15] Xie, Y., Cao, S., Dong, H., Li, Q., Chen, E., Zhang, W., et al. (2020) Effect of Regular Intravenous Immunoglobulin Therapy on Prognosis of Severe Pneumonia in $\mathrm{Pa}$ tients with COVID-19. Journal of Infection, 81, 318-356.

https://doi.org/10.1016/j.jinf.2020.03.044

[16] Zantah, M., Castillo, E.D., Gangemi, A.J., Patel, M., Chowdhury, J., Verga, S., et al. (2020) Anakinra and Intravenous IgG versus Tocilizumab in the Treatment of COVID-19 Pneumonia. medRxiv. [Preprint] https://doi.org/10.1101/2020.09.11.20192401

[17] Cao, W., Liu, X., Bai, T., Fan, H., Hong, K., Song, H., et al. (2020) High-Dose Intravenous Immunoglobulin as a Therapeutic Option for Deteriorating Patients with Coronavirus Disease 2019. Open Forum Infectious Diseases, 7, ofaa102. https://doi.org/10.1093/ofid/ofaa102

[18] Mohtadi, N., Ghaysouri, A., Shirazi, S., Ansari, S., Shafiee, E., Bastani, E., et al. (2020) Recovery of Severely Ill COVID-19 Patients by Intravenous Immunoglobulin 
(IVIG) Treatment: A Case Series. Virology, 548, 1-5.

https://doi.org/10.1016/j.virol.2020.05.006

[19] Sakoulas, G., Geriak, M., Kullar, R., Greenwood, K.L., Habib, M., Vyas, A., et al. (2020) Intravenous Immunoglobulin (IVIG) Significantly Reduces Respiratory Morbidity in COVID-19 Pneumonia: A Prospective Randomized Trial. medRxiv. [Preprint] https://doi.org/10.1101/2020.07.20.20157891

[20] Schwaiger, J., Karbiener, M., Aberham, C., Farcet, M.R, Kreil, T.R., et al. (2020) No SARS-CoV-2 Neutralization by Intravenous Immunoglobulins Produced from Plasma Collected before the 2020 Pandemic. The Journal of Infectious Diseases, 222, 1960-1964. https://doi.org/10.1093/infdis/jiaa593

[21] Cheng, M.H., Zhang, S., Porritt, R.A., Noval Rivas, M., Paschold, L., Willscher, E., et al. (2020) Superantigenic Character of an Insert Unique to SARS-CoV-2 Spike Supported by Skewed TCR Repertoire in Patients with Hyperinflammation. Proceedings of the National Academy of Sciences of the United States of America, 117, 25254-25262. https://doi.org/10.1073/pnas.2010722117

[22] Díez, J.M., Romero, C. and Gajardo, R. (2020) Currently Available Intravenous Immunoglobulin Contains Antibodies Reacting against Severe Acute Respiratory Syndrome Coronavirus 2 Antigens. Immunotherapy, 12, 571-576.

https://doi.org/10.2217/imt-2020-0095

[23] Callard, F. and Perego, E. (2021) How and Why Patients Made Long Covid. Social Science \& Medicine, 268, Article ID: 113426. https://doi.org/10.1016/j.socscimed.2020.113426

[24] Davis, H.E., Assaf, G.S., McCorkell, L., Wei, H., Low, R.J., Re'em, Y., et al. (2020) Characterizing Long COVID in an International Cohort: 7 Months of Symptoms and Their Impact. medRxiv. [Preprint] https://doi.org/10.1101/2020.12.24.20248802

[25] Sudre, C.H., Murray, B., Varsavsky, T., Graham, M.S., Penfold, R.S., Bowyer, R.C., et al. (2020) Attributes and Predictors of Long-COVID: Analysis of COVID Cases and Their Symptoms Collected by the Covid Symptoms Study App. medRxiv. [Preprint] https://doi.org/10.1101/2020.10.19.20214494

[26] Michelen, M., Manoharan, L., Elkheir, N., Cheng, V., Dagens, D., Hastie, C., et al. (2020) Characterizing Long-Term Covid-19: A Rapid Living Systematic Review. medRxiv. [Preprint] https://doi.org/10.1101/2020.12.08.20246025

[27] Younger, D.S. (2020) Book Review: I-Cubed and the Autoimmune Brain: A Five-Step Plan. World Journal of Neuroscience, 10, 29-36. https://doi.org/10.4236/wjns.2020.101005

[28] Henderson, L.A., Canna, S.W., Friedman, K.G., Gorelik, M., Lapidus, S.K., Bassiri, H., et al. (2021) American College of Rheumatology Clinical Guidance for Multisystem Inflammatory Syndrome in Children Associated With SARS-CoV-2 and Hyperinflammation in Pediatric COVID-19: Version 2. Arthritis \& Rheumatology, 73, e13-e29. https://doi.org/10.1002/art.41616

[29] Newburger, J.W., Takahashi, M., Gerber, M.A., Gewitz, M.H., Tani, L.Y., Burns, J.C., et al. (2004) Diagnosis, Treatment, and Long-Term Management of Kawasaki Disease: A Statement for Health Professionals from the Committee on Rheumatic Fever, Endocarditis and Kawasaki Disease, Council on Cardiovascular Disease in the Young, American Heart Association. Circulation, 110, 2747-2771. https://doi.org/10.1161/01.CIR.0000145143.19711.78

[30] Younger, D.S., Mast, P.A. and Bouboulis, D.A. (2016) PANDAS: Baseline Immunoglobulin Levels Predict Achievement of Remission at One Year Following IVIg Therapy. Journal of Neurology and Neurosurgery, 3, Article No. 122. 
[31] Ballow, M. (2002) Primary Immunodeficiency Disorders: Antibody Deficiency. Journal of Allergy and Clinical Immunology, 109, 581-591. https://doi.org/10.1067/mai.2002.122466

[32] Shields, A.M., Burns, S.O., Savic, S. and Richter, A.G. (2021) UK PIN COVID-19 Consortium. COVID-19 in Patients with Primary and Secondary Immunodeficiency: The United Kingdom Experience. Journal of Allergy and Clinical Immunology, 147, 870-875.e1. https://doi.org/10.1016/j.jaci.2020.12.620

[33] Younger, D.S. (2020) Post-Infectious Sequela of SARS-COV-2 Infection in Adults and Children: An Overview of Available Agents and Clinical Responsiveness. Archives of Neurology \& Neurological Disorders, 3, e102.

[34] Younger, D.S. (2016) The Human Microbiome and I-Cubed: A Modern Medical Paradigm. World Journal of Neuroscience, 6, 260-286.

https://doi.org/10.4236/wjns.2016.64031

[35] Smatti, M.K., Cyprian, F.S., Nasrallah, G.K., Al Thani, A.A., Almishal, R.O. and Yassine, H.M. (2019) Viruses and Autoimmunity: A Review on the Potential Interaction and Molecular Mechanisms. Viruses, 11, Article No. 762.

https://doi.org/10.3390/v11080762

[36] Rodríguez, Y., Novelli, L., Rojas, M., De Santis, M., Acosta-Ampudia, Y., Monsalve, D.M., et al. (2020) Autoinflammatory and Autoimmune Conditions at the Crossroad of COVID-19. Journal of Autoimmunity, 114, Article ID: 102506. https://doi.org/10.1016/j.jaut.2020.102506

[37] Alberti, P., Beretta, S., Piatti, M., Karantzoulis, A., Luisa Piatti, M., Santoro, P., et al. (2020) Guillain-Barré Syndrome Related to COVID-19 Infection. Neurology: Neuroimmunology \& NeuroInflammation, 7, e741. https://doi.org/10.1212/NXI.0000000000000741

[38] Nathan, N., Prevost, B. and Corvol, H. (2020) Atypical Presentation of COVID-19 in Young Infants. Lancet, 395, 1481. [Correction: Lancet, 2020, 396, 668] https://doi.org/10.1016/S0140-6736(20)30980-6

[39] Merrill, J.T., Erkan, D., Winakur, J. and James, J.A. (2020) Emerging Evidence of a COVID-19 Thrombotic Syndrome Has Treatment Implications. Nature Reviews Rheumatology, 16, 581-589. https://doi.org/10.1038/s41584-020-0474-5

[40] Younger, D.S. (2021) Post-Acute Sequelae of SARS-Cov-2 Infection (PASC): Peripheral, Autonomic and Central Nervous System Features In a Child. Neurological Sciences, In Press. 